Materials chemistry and physics 142, $469-478$ (2013)

\title{
Lightweight Nanocomposites based on Poly(Vinylidene Fluoride) and Al Nanoparticles: Structural, Thermal and Mechanical Characterization and EMI Shielding Capability
}

\author{
J. Arranz-Andrés ${ }^{a, *}$, N. Pulido-González ${ }^{a}$, C. Fonseca ${ }^{b}$, E. Pérez a, M. L. Cerrada a \\ a Instituto de Ciencia y Tecnología de Polímeros (ICTP-CSIC), Juan de la Cierva 3, 28006 Madrid, Spain \\ b POLCA, Departamento de Química Industrial y Polímeros, E. T. de Ingenieros Industriales, Universidad \\ Politécnica de Madrid, Ronda de Valencia, 3. 28012 Madrid, Spain.
}

\begin{abstract}
Novel (nano)composites based on PVDF and different content of Al nanoparticles have been prepared in order to learn about their electromagnetic interference shielding capability. Very promising results are obtained, with an excellent balance between shielding and sample weight, so that these materials are potentially good alternatives to replace neat metals for that application. Moreover, a complete structural and morphological characterization, as well as an evaluation of their thermal and mechanical behavior, has been also performed.
\end{abstract}

Keywords: Composite materials, X-ray diffraction, mechanical properties, indentation

${ }^{*}$ Corresponding author: J. Arranz-Andrés

Phone: +34-91-2587416, Fax: +34-91-5644 853

E-mail: jarranz@ictp.csic.es 


\section{Introduction}

There are currently many concerns and more chances of deterioration of the electromagnetic wave environment, known as electromagnetic interference $(E M I)$, due to the increase in use of high operating frequency and bandwidth in electronic systems. This EMI has adverse effects on electronic equipments, such as false operation, because of unwanted electromagnetic waves and leakage of information in wireless telecommunications [1-4]. Hence, in order to maintain the electromagnetic compatibility of the end product, lightweight EMI shielding materials are required to sustain the good working environment of the devices. On the other hand, there is an increasing concern about the biological and health effects of electric and magnetic fields [5-7], and these lightweight EMI shielding materials could be a suitable solution in many circumstances.

EMI shielding refers to the reflection, absorption or multiple reflection of the electromagnetic radiation by a shielding material, which thereby acts as a shield against the penetration of the radiation through it [3]. Metals and metallic compounds are conventionally used as EMI shielding materials as they have high shielding efficiency owing to their good electrical conductivity. They exhibit, however, poor chemical resistance, oxidation, corrosion, high density, and difficulty in processing. An interesting approach for overcoming these limitations is the development of matrix composites. These are typically metal-polymer blends utilizing metal or metallic particles as a dispersed phase into a polymeric matrix. These composites are more flexible and lighter, combining the processability of polymers with the high shielding efficiency of metal particles.

Though this approach is promising, it shows several important drawbacks. First, dispersed particles larger than a few hundred nanometers can induce "stress concentrators" in the matrix, leading to fatigue and early failure. Second, the host materials and metallic phases might tend to detach under different conditions and, then, inhomogeneities in the final composites may be found. Finally, there is a question of miscibility: dispersions of large particles are difficult to process. These shortcomings might disappear if metal nanoparticles are used instead of conventional microsized ones and formation of nanoparticle aggregates is skipped during processing. The embedding of nanoscopic metal structures into polymeric matrices might favor nanoparticle-polymer interactions. Accordingly, a lower content of nanoparticles could be, then, necessary to lead to similar or even improved properties, avoiding problems during processing and leading to lighter ultimate (nano)composites. 
A variety of materials can be used for EMI shielding. However, the EMI shielding characteristic of the candidate materials should not be the only property considered in the design of electronic enclosures and there are other structural, thermal, and electrical properties, i.e., density, strength and stiffness, which should be taken into account by designers. On one hand, poly(vinylidene fluoride), PVDF, is a very good option as polymeric matrix because it shows excellent mechanical properties, high continuous use of temperature and strong piezoelectricity. These features make possible various scientific and industrial applications, additionally to those related to antistatic shielding, as an ultrasound transducer in non-destructive evaluation and medical ultrasound [8-10], and for self-regulated heaters, current protectors, and conducting electrodes for lithium batteries. It is, therefore, a very promising polymeric matrix in the conductive (nano)composite field. On the other hand, metal candidates concern aluminum, brass, bronze, copper, gold, lead, nickel, silver, tin, and stainless steel. The metal density and thermal conductivity are critical parameters, while cost and stiffness are also very important aspects. Nevertheless, their EMI shielding is not so important due to the fact that the thickness that is required for structures is usually enough for effective shielding. Therefore, EMI properties are considered to have the same importance level as cost and stiffness.

The current investigation is focused on novel lightweight hybrids based on PVDF and Al nanoparticles. Aluminum is selected because of its remarkable low density and its ability to resist corrosion. The use of nanoparticles instead of microsized particles would permit incorporation of lower contents, if Al agglomerates are not generated during manufacture. The preparation of these PVDF/Al (nano)composites has been performed by melt compounding, because it allows using conventional plastics extrusion molding equipments, with the subsequent environmental and economical advantages in terms of absence of solvents during processing. In addition, their phase transitions and other structural, morphological and mechanical characteristics have been analyzed in order to determine if these materials meet mandatory requirements for their further applicability. The shielding response of these hybrids to high-energy radiation is also preliminarily evaluated at the synchrotron radiation wavelength. Several techniques have been then employed, these including wide-angle X-ray diffraction, with either conventional or synchrotron radiation, and ATR Fourier infrared for structural details; scanning electron microscopy, SEM, for morphological aspects; differential scanning calorimetry, DSC, for phase transitions; microhardness and uniaxial stress-strain measurements for mechanical response; and X-ray diffraction with synchrotron radiation for shielding to electromagnetic interference. 


\section{Experimental Part}

\subsection{Materials}

A commercially available poly(vinylidene fluoride), PVDF, with trade name of Kynar 741 (kindly supplied by Arkema), has been used in the present research. Aluminum, Al, nanoparticles (purchased to IOLITEC) with an average particle size of $18 \mathrm{~nm}$, have been employed in the present research.

\subsection{Hybrid and Film Preparation}

Composites with different contents in Al nanoparticles $(1,2,5,8,10,15,20 \%$ in volume), labeled as Al1, Al2, Al5, Al8, Al10, Al15 and Al20 respectively, were prepared through melt processing at $190{ }^{\circ} \mathrm{C}$ and at $120 \mathrm{rpm}$ for $40 \mathrm{~min}$ in a Haake Minilab (Thermo Electron Corporation) twin-screw extruder, with a volumetric capacity of $7 \mathrm{~cm}^{3}$, using corotating conical screws.

After extrusion of the two components, films with similar thickness were obtained by compression molding in a Collin press between hot plates $\left(210^{\circ} \mathrm{C}\right)$ at pressures from 2 to $6 \mathrm{MPa}$ for $5 \mathrm{~min}$. A relatively fast cooling (around $80^{\circ} \mathrm{C} / \mathrm{min}$ ) between plates of the press refrigerated with cold water was applied to the different films from the melt to room temperature.

\subsection{Sample Characterization}

Wide-angle X-ray diffraction (WAXS) patterns were recorded at room temperature in the reflection mode by using a Bruker D8 Advance diffractometer provided with a PSD Vantec detector (from Bruker, Madison, Wisconsin). Cu Ka radiation $(\lambda=0.1542 \mathrm{~nm}$ ) was used, operating at $40 \mathrm{kV}$ and $40 \mathrm{~mA}$. The parallel beam optics was adjusted by a parabolic Göbel mirror with horizontal grazing incidence Soller slit of $0.12^{\circ}$ and LiF monochromator. The equipment was calibrated with different standards. A step scanning mode was employed for the detector. The diffraction scans were collected within the range of $2 \theta=3-60^{\circ}$, with a $2 \theta$ step of $0.024^{\circ}$ and $0.2 \mathrm{~s}$ per step.

All the samples were also studied at room temperature using synchrotron radiation (with $\lambda=0.150$ $\mathrm{nm}$ ) on the soft-condensed matter A2 beamline at DESY (Hamburg, Germany). A MARCCD detector, placed at $135 \mathrm{~mm}$ from the sample, was used. The calibration was performed with the crystalline diffractions of polyethylene terephthalate. The 2D X-ray diffractograms were processed using the A2 tool program 
developed at DESY to support beamline A2 data processing. The transmission of the samples to this radiation was determined by measuring the relative intensities of the direct beam before and after the sample (in the ionization chamber and in the beamstop, respectively).

Additionally, variable-temperature experiments under real-time conditions using synchrotron radiation were also performed in beamline A2 at DESY for the PVDF both in the small and wide angle regions, SAXS and WAXS, respectively. The experiments comprise the heating from 26 up to $190{ }^{\circ} \mathrm{C}$ at 8 ${ }^{\circ} \mathrm{C} \cdot \mathrm{min}^{-1}$. The profiles were normalized to the primary beam intensity and the background from an empty sample was subtracted. The data acquisition was done in frames of $15 \mathrm{~s}$. A sample of crystalline PET was used for WAXS calibration, and the different orders of the long spacing of rat-tail cornea $(\mathrm{L}=65 \mathrm{~nm})$ were utilized for the SAXS detector.

The IR spectra were obtained on films using a Perkin Elmer FTIR spectrometer equipped with an ATR device, scanning between 650 and $4000 \mathrm{~cm}^{-1}$. Sixty four scans were accumulated for each specimen, at a resolution of $4 \mathrm{~cm}^{-1}$.

Scanning electron microscopy (SEM) experiments were carried out with a XL30 ESEM PHILIPS equipment. The samples were cryo-fractured prior to SEM observations.

Calorimetric analyses were carried out in a TA Instruments Q100 calorimeter connected to a cooling system and calibrated with different standards. The sample weights ranged from 7 to $10 \mathrm{mg}$ and the heating rate used was $20^{\circ} \mathrm{C} \cdot \mathrm{min}^{-1}$. For crystallinity determinations, a value of $104.5 \mathrm{~J} \mathrm{~g}^{-1}$ has been taken as enthalpy of fusion of a perfect crystalline material $[11,12]$. On the other hand, the glass transition temperature, $T_{g}$, was determined as the temperature where the specific heat increment is half the total one at the transition.

Dumb-bell shaped specimens with gauge dimensions $15 \mathrm{~mm}$ in length and $1.9 \mathrm{~mm}$ in width were punched out from the sheets with a standardized die. Tensile testing was carried out using an Instron Universal testing machine calibrated according to standard procedures. All of the specimens were drawn at a crosshead speed of $10 \mathrm{~mm} \cdot \mathrm{min}^{-1}$ at $23^{\circ} \mathrm{C}$. The different mechanical parameters were calculated from the stress-strain curve. At least four specimens were tested for each material and the mean values were reported. The uncertainty in these mean values was less than $10 \%$. 
A Vickers indentor attached to a Leitz microhardness $(\mathrm{MH})$ tester was used to perform microindentation measurements. The experiments were carried out at $23{ }^{\circ} \mathrm{C}$, with contact load of $0.98 \mathrm{~N}$ for $25 \mathrm{~s} . \mathrm{MH}$ values $(\mathrm{MPa})$ were calculated according to the following relationship [13, 14]:

$$
M H=2 \sin 68^{\circ}\left(P / d^{R}\right)
$$

where $\mathrm{P}(\mathrm{N})$ is the contact load and $\mathrm{d}(\mathrm{mm})$ is the diagonal length of the projected indentation area.

\section{Results and discussion}

\subsection{Structural and morphological characterization}

Figure 1 shows the WAXS profiles at room temperature for PVDF and the different hybrids with Al nanoparticles, their semicrystalline nature being clearly noticeable. PVDF is characterized by its polymorphism, i.e., the possibility to form different crystalline structures. This polymer may present, at least, four different well-known crystalline structures depending on the crystallization conditions: the $\alpha, \beta, y$ and $\delta$ phases [15]. The non-polar a phase, most readily obtained from the melt, has a trans-gauche conformation (TG+TG-). The polar $\beta$ phase, with improved pyro and piezoelectric properties, has an all trans planar zigzag conformation (TTT). The polar y phase, obtained from high-temperature crystallization, has a conformation intermediate between that of the $\alpha$ and the $\beta$ phases, consisting of a sequence of three trans linked to a gauche (TTTG-TTTG'). The $\delta$ phase is a polar version of the $\alpha$ phase and is obtained by polarizing an originally $\alpha$-phase sample in a high electric field [16]. The diffractions observed in Figure 1 are those characteristics from the a polymorph, as expected, since films were prepared by a relatively fast cooling from the melt. This form exhibits four main diffractions $[17,18]$ assigned to the following Miller indices: (100), (020), (110) and (021). A significant reduction of normalized intensity values as well as a broadening of these peaks is revealed as Al nanoparticles are incorporated to the resulting materials. These features mean that although the crystalline character is kept in the hybrids materials, some changes are occurring within PVDF crystalline regions because of the Al incorporation. Evidently, the Al reflections (at $2 \theta$ values of 38.5 and 44.7 degrees) are also observed, with intensity proportional to the Al content in the hybrids.

The almost unique existence of the $\alpha$ crystalline form in the PVDF homopolymer and its Al hybrids can be also deduced from the FTIR spectra in the region ranging from 700 to $1000 \mathrm{~cm}^{-1}$, since the crystalline phases of PVDF can be examined from the infrared absorption bands [19-21] between 400 and $1000 \mathrm{~cm}^{-1}$. 
The upper plot in Figure 2 shows the presence of the band at $873 \mathrm{~cm}^{-1}$, which is attributed to the amorphous phase of PVDF independently of the crystalline phase developed, and of the bands at 764, 796 and $976 \mathrm{~cm}^{-1}$ that are associated with the a phase of PVDF. These results are in agreement with those aforementioned from X-ray experiments. Nevertheless, the presence of bands at 840 and $1276 \mathrm{~cm}^{-1}$ in either the PVDF homopolymer or the different nanocomposites may indicate the presence of the $\beta$ polymorph, although in a very small content, non-detectable by $\mathrm{X}$-ray measurements.

Besides the assessment of the almost exclusive generation of the $\alpha$ polymorph under the experimental conditions used, FTIR spectra prove also the existence of certain interactions between the PVDF matrix and the Al nanoparticles. Thus, a shift to lower wave numbers is seen as Al content increases in the hybrid, as depicted in Figure 2 (upper and lower plots). Moreover, variations in width and definition of the peaks are also noticed with rising Al concentration. These changes are seen, for instance, in the peaks observed at 976 and $764 \mathrm{~cm}^{-1}$. The $976 \mathrm{~cm}^{-1}$ band corresponds to the $\mathrm{CH}_{2}$ twisting vibration while $764 \mathrm{~cm}^{-1}$ is arising from the $\mathrm{CF}_{2}$ bending and from the skeletal $\mathrm{CF}-\mathrm{CH}-\mathrm{CF}$ bending [22]. The disturbance of those two absorption peaks in the FTIR spectra of the polymer nanocomposite seems to suggest the possible electrostatic interaction between the CF dipoles in the polymer chain and the surface charge on nanoparticles and, accordingly, an electrical coupling that hinders the otherwise free rotation of different types of bonds $[23,24]$.

One of the main issues associated with manufacture of melt compounded (nano)composites is the difficulty of achieving a good distribution of the nanofiller in the matrix without formation of large size aggregates. A poor compatibility between the thermoplastic PVDF matrix and Al nanoparticles in the melt state would turn out in weak polymer-nanofiller interactions and would complicate their further dispersion, resulting in their agglomeration and, consequently, poor matrix-filler adhesion. As just mentioned, FTIR results have confirmed the existence of interactions between the PVDF and the AI nanoparticles, possibly electrostatic-like interactions from polymeric dipoles to the surface charge on nanoparticles. These can contribute to favor the adhesion at PVDF-Al interfaces and facilitate the homogenous Al dispersion within the PVDF matrix. SEM pictures were obtained and used to evaluate the Al nanoparticles distribution and, accordingly, homogeneity within the hybrid materials. Figure 3 primarily provides evidence of high degree of the Al dispersion and distribution within the PVDF polymeric matrix. Absence of detectable metallic domains of large size across the film is deduced even at the highest Al contents, although some Al agglomerates 
have been generated. The average size of these Al aggregates, independently of the Al content, is ranging from around 40 to $175 \mathrm{~nm}$, fact that indicates the importance of the existing PVDF-Al interactions and the efficiency of the melt preparation method.

\subsection{Thermal transitions}

Additional information of the crystalline structure in these hybrids can be attained from calorimetric results. Figure 4 shows the melting and crystallization processes in upper and lower plot, respectively, for the different samples. An endothermic peak ascribed to the melting process of the PVDF crystallites is exhibited, as seen in Figure 4a. As aforementioned, the a polymorph is practically the unique one developed in these quenched specimens, as deduced from WAXS profiles and FTIR spectra. However, two melting processes seem to be merged in the neat homopolymer and in its nanocomposites. These features seem to be related to melting-recrystallization phenomena of these a crystals, which become so important at high temperatures allowing formation of thicker crystallites from those initially smaller and less compact ones. However, data from the literature [25] support the idea that, sometimes, the double melting peaks observed in the DSC experiment on PVDF are because of the polymorphic structure peculiar to this polymer. In particular, this reference indicated that the low temperature melting peak, around $170{ }^{\circ} \mathrm{C}$, is due to the melting of $\alpha$-form crystals, while the high temperature peak, located at about $180{ }^{\circ} \mathrm{C}$, is associated with the $\beta$-form. This second alternative seems not to be plausible in these specimens under study because of their small $\beta$ form content, undetectable by WAXS and with FTIR bands of small intensity. The only possibility might be a phase transition from the a polymorph, after its melting, to the $\beta$-form. Figure $5 \mathrm{a}$, which represents the variable-temperature diffraction patterns found in PVDF during its melting process, shows that this transformation does not take place.

Moreover, the real-time variable-temperature Lorentz-corrected SAXS profiles corresponding to this PVDF melting are displayed in Figure 5b. A clear long spacing is seen, this peak undergoing an important shift to lower $s$ values with temperature, associated with the improvement of the PVDF crystalline morphology and, then, the thickening of its crystallites. Consequently, considerable melting-recrystallization phenomena are occurring during the melting process.

Further information can be obtained from these profiles by analysis of the relative SAXS invariant. This is a magnitude used in the lamellar stack model theory of semicrystalline polymers, which is directly 
related to the electron density differences between the two phases (amorphous and crystalline), so that it has rather important variations during crystallization, recrystallization or melting processes in semicrystalline polymers [26-28]. The present results (see upper plot in Figure 5c) prove the existence of different regions during melting of PVDF: an initial one, up to around 45-50 ${ }^{\circ} \mathrm{C}$, where an almost constancy of the SAXS invariant is seen; a second zone, between $50^{\circ} \mathrm{C}$ to around $150{ }^{\circ} \mathrm{C}$, with an important increase of the invariant; and a final one characterized by a primary and sharp decrease, related to the main melting process. Its exact location can be determined from its derivative.

These distinct regions can also be observed from the values of the most probable long spacing as function of temperature, deduced from the Lorentz-corrected SAXS profiles, as observed in the lower plot of Figure $5 \mathrm{c}$. The first one, up to around $50 \stackrel{\circ}{\circ}$, where the long spacing is almost constant; a second region, between $50 \stackrel{\circ}{\mathrm{C}}$ to around $150 \stackrel{\circ}{\circ}$, with a moderate increase of $\mathrm{L}$; and a final one, with a very important thickening, this being ascribed to significant melting-recrystallization phenomena. All of these results confirm the accuracy on the assumption of successive melting-recrystallization processes triggering the two DSC melting peaks during the melting process in these PVDF-Al nanocomposites.

These very important recrystallization phenomena are also similarly observed in the different hybrids, with subtle variations in relation to PVDF.

In relation to melting and glass transition temperatures, they remain rather unvarying, independently of presence and content of Al nanoparticles within PVDF. Temperature of crystallization does not change much either, although a slight nucleating effect is seen at the highest Al concentrations and, then, this transition is shifted a little to higher temperatures (see data in Table 1). A larger variation is observed in the degree of PVDF crystallinity by Al presence and an opposite tendency is found in those Al15 and Al20 nanocomposites if initial melting or crystallization is considered. Accordingly, a significant increase of crystallinity is seen during first melting while a reduction is observed when crystallization takes place. As aforementioned, Al nanoparticles seem to act as nucleating agents in those hybrids with the highest $\mathrm{Al}$ contents and if samples are left enough time at room temperature after processing, as occurred before performing DSC experiments, crystallinity can be increased although crystallites could need longer times to grow during cooling process. 


\subsection{Mechanical response}

(Nano)composites with advanced properties (conductive, antimicrobial, electronic, etcetera) must accomplish a minimum mechanical performance for their ultimate applicability. None material will be commercialized if these requirements are not attainable, independently of the goodness of their specific functionality. Homogenous nanoparticles distributions within the polymeric matrix and polymer-nanoparticle interactions are of primary importance in the resulting mechanical behavior and they are crucial factors to achieve a strong mechanical reinforcement effect.

Figure 6 depicts the results obtained from microhardness, $\mathrm{MH}$, measurements as evaluation of surface mechanical behavior in these hybrids. $\mathrm{MH}$ examines primarily the resistance of the polymer to plastic deformation, providing an idea about local strain. However, several effects can be distinguished in these measurements [14]: an elastic deformation, a permanent plastic one and a viscoelastic time-dependent contribution. Therefore, though microhardness measurements are performed at the surface of films and, consequently, are related to the superficial mechanical response, they are also associated with other bulk properties, as the elastic modulus. Moreover, its determination has important advantages: its quickness, the small amount of material required, and its use as a rapid and reliable method of determining inhomogeneities caused during the processing of polymers and composites materials [29, 30]. Accordingly, several $\mathrm{MH}$ measurements were performed at different sections within the films to check the Al distribution homogeneity and to evaluate the mechanical behavior. Regarding the first aspect, all of the nanocomposites were found to be rather homogeneous, corroborating the information achieved from SEM pictures.

In addition, Figure 6 clearly shows an important $\mathrm{MH}$ increase as $\mathrm{Al}$ nanoparticles concentration is raised in the hybrids. The rigidity changes considerably with the incorporation of the metallic nanoparticles, leading to a substantial strengthening compared with neat PVDF. This stiffness boost is attributed to two facts: on one hand, the reinforcement effect of the nanofiller and, on the other hand, to the increase in crystallinity observed for those materials with the highest Al contents.

Stress-strain measurements have been carried out to get a deeper information on the mechanical performance of these nanocomposites and to know the correlation between the surface and bulk rigidity. Figure 7 shows the stress-strain curves for the different materials under analysis and several features can be deduced from them as Al content is raised. First of all, a clear increase in stiffness (from the slope of the 
elastic initial deformation region) (see results in Table 2 and Figure 8); secondly, a rise in the yield stress value; and, finally, shorter deformations at break (and much reduced toughness). Then, a ductile-brittle transition in how deformation takes place is promoted by Al incorporation. Pristine PVDF matrix shows a regular stretching process with necking formation, characteristic in semicrystalline polymers drawn at temperatures well below their melting, while the deformation mechanism in the nanocomposites is exchanging to much fragile with increasing nanoparticle content. These differences in the mechanical response are also related to the elongation at break and yield stress, as observed in Figure 7 and Table 2 . The Al nanoparticle domains interrupt the deformability capacity of PVDF and provoke its early breakdown and, hence, the decrease in the rupture strain.

The variation of Young's modulus with Al content is represented in Figure 8. Overall rigidity rises considerably with the incorporation of the Al nanoparticles, as observed previously for that magnitude at the surface (through $\mathrm{MH}$ results). Rather homogeneous $\mathrm{Al}$ nanoparticle dispersions, non-existence of $\mathrm{Al}$ agglomerates of large size and some PVDF-Al interactions have been found (already commented). This fact, together with the crystallinity boost observed as increasing Al content in these hybrids, leads to this stiffness improvement and to change in deformation mechanism compared with neat PVDF. Nevertheless, the enhancement is not as large as expected when considering a perfect mixing law, taking into consideration that aluminum exhibits a modulus value of around $70 \mathrm{GPa}$. This might be attributed to the fact that the Al aspect ratio is around 1 when this metal is in a nanoparticle spherical shape and, then, its reinforcing capacity is not as efficiently transferred to the polymeric matrix as if Al would be in a fiber-like of ribbon-like shape, for identical dispersion and interactions.

Young's modulus dependence on Al composition is similar to that observed in microhardness. This behavior is in agreement with that proved by some other polymeric materials reported in the literature [14, 31-36] and it is fulfilled by different systems with $\mathrm{MH}$ and $\mathrm{E}$ values within a very broad range: from thermoplastic elastomers to very rigid polymers. Accordingly, a linear dependence, between elastic modulus and $\mathrm{MH}$ in the double log-log scale was proposed [14], here represented in the inset of Figure 8:

$$
\mathrm{MH}=\mathrm{a} \mathrm{E}^{\mathrm{b}}
$$

where $\mathrm{a}$ and $\mathrm{b}$ are constants. The corresponding values for $a$ and $b$ are 1.34 and 0.63 , respectively. 


\subsection{Electromagnetic interference shielding}

Metals and their composites are suitable materials to shield electromagnetic interference, EMI [37], although they present several disadvantages, such as high weight, poor processability and corrosive susceptibility. Metal coatings are, therefore, commonly used for shielding, but they still suffer wear and poor scratch resistance. Reinforced polymer composites could be the best alternative to replace metals. A great deal of research has been conducted on the use of polymeric composites for EMI shielding applications. For instance, carbon-black and carbon-fiber filled ethylene-vinyl acetate copolymer and natural rubber were prepared [38], studying their potential applications for EMI shielding in both the microwave (100-2000 MHz) and $\mathrm{X}$-band region $(8-12 \mathrm{GHz})$. They reported that the composites with fiber loading greater than 20 parts per hundred parts of rubber can be used for EMI shielding with shielding effectiveness (SE) of more than 20 dB. Similarly, a frequency independent SE behavior has been reported for CNF-reinforced polystyrene composites [39]. The study demonstrated that the loading of $20 \mathrm{wt} \%$ of nanofibers resulted in a SE value of $30 \mathrm{~dB}$.

For the present hybrids, we have tested their EMI shielding capability by acquiring the transmission X-ray diagrams by using synchrotron radiation with a wavelength of $0.150 \mathrm{~nm}$. Figure 9 shows the 2D pictures for PVDF and the Al15 hybrid. It can be observed that the signal intensity (and the total intensity reaching the detector) decreases very much as the Al content increases, in such a way that for Al15 the signal is already very small, compared with neat PVDF.

Figure 10 represents a quantification of the intensity that reaches the CCD detector after normalization to primary beam intensity, thickness and acquisition time. A decrease of the intensity signal is clearly observed as Al nanoparticles content rises in the hybrid at this wavelength.

EMI shielding effectiveness (SE) from attenuation upon transmission has been tentatively evaluated at the frequency of the used synchrotron radiation (2 EHz). SE (expressed in decibels) is defined as function of the ratio of radiation transmitted in presence of a given material, $E_{0}$, and without the material present, $E_{1}$ [3]:

$$
S E(\mathrm{~dB})=20 \log E_{0} / E_{1}
$$


The SE results for the different samples are represented in Figure 10, indicating that values above $19 \mathrm{~dB}$ are obtained in Al15 and hybrids with highest Al incorporation. It seems that a plateau region is reached once Al content is high enough and no further increase is achieved. Nevertheless, it should be mentioned that a SE of $20 \mathrm{~dB}$ provides $90 \%$ attenuation of the electromagnetic radiation.

From the attenuation coefficient reported by B.L. Henke et al.[40] for an X-ray energy of $8265 \mathrm{eV}(\lambda$ $=1.5 \AA, 2 \mathrm{EHz})$, the SE value for metallic aluminum at the same frequency $(2 \mathrm{EHz})$ and thickness $(1 \mathrm{~mm})$ is determined to be $103.9 \mathrm{~dB}$. It is deduced, therefore, that the Al20 hybrid shows a reduction in shielding capability of around $80 \%$ in relation to neat $\mathrm{Al}$, counterbalanced by a $60 \%$ decrease in weight. Moreover, the Al10 specimen exhibits a similar reduction in electromagnetic attenuation but a much considerable weight drop (higher than $80 \%$ ). Consequently, these hybrids represent a good compromise between shielding and weight (and price).

It should be mentioned that if greater values of EMI shielding are required, Al can be replaced by $\mathrm{Cu}$. Thus, PVDF based hybrids that incorporate $\mathrm{Cu}$ nanoparticles show values of around $68 \mathrm{~dB}$ for the hybrid with a 20 vol.\%)[41], although that material is about $35 \%$ heavier than Al20 nanocomposite. A balance attenuation / weight should be analyzed to meet essential requirements at a given application.

More complete EMI shielding measurements on the present Al hybrids are under progress, but these results are really promising ones for the materials here prepared and evaluated.

\section{Conclusions}

Novel hybrids based on PVDF and different contents of Al nanoparticles have been prepared. They exhibit a semicrystalline character although the Al incorporation leads to some changes within PVDF crystalline regions. Thus, crystallinity is increased for the highest Al contents, Al15 and Al20 samples. Moreover, the crystallization temperature is moved to slightly higher values, indicating the nucleation effect of high nanoparticles contents. On the other hand, the FTIR spectra show a shift of several bands, indicating the existence of interactions between the PVDF matrix and the Al nanoparticles.

Microhardness measurements reveal that rigidity increases as Al nanoparticles concentration is raised in the hybrids. These results are in a good agreement with those obtained from stress-strain tests. 
Then, stiffness is boosted as Al content raises because of the inherent reinforcement effect of nanofiller and the increase in PVDF crystallinity.

Finally, EMI shielding effectiveness from attenuation upon transmission has been evaluated for the frequency of the used synchrotron radiation $(2 \mathrm{EHz})$. The results found are promising since values of around $20 \mathrm{~dB}$, which provides at least a $90 \%$ attenuation of the electromagnetic radiation, are reached by Al15 and hybrids with higher Al content, with a good balance between shielding and sample weight. Therefore, these materials are potentially a good alternative to replace neat metals for EMI shielding.

\section{Acknowledgements}

Financial support of Ministerio de Ciencia e Innovación, MICINN (project MAT2010-19883) is acknowledged. J. Arranz-Andrés is grateful to the CSIC JAE-Doc Program for the financial support. The synchrotron work leading to these results was performed at DESY and received funding from the European Community's Seventh Framework Programme (FP7/2007-2013) under grant agreement $n^{\circ} 312284$. We are grateful for collaboration of the Hasylab personnel, especially Dr. S.S. Funari.

\section{References}

[1] P. Cylayton R, Introduction to Electromagnetic Compatibility, Second ed., John Wiley \& Sons Inc., New Jersey, 2006.

[2] K. Malaric, EMI Protection for Communication Systems, Artech House, 2010.

[3] X.C. Tong, Advanced Materials and Design for Electromagnetic Interference Shielding, CRC Press, New York, 2009.

[4] S. Celozzi, R. Araneo, G. Lovat, Electromagnetic Shielding, John Wiley \& Sons, Inc, Hoboken, New Jersey, 2008.

[5] ICNIRP, Exposure to High Frequency Electromagnetic Fields, Biological Effects and Health Consequences (100 kHz-300 GHz), ICNIRP, Munich, 2009.

[6] ICNIRP, Health Physics, 74 (1998) 494-522.

[7] ANSI, Safety levels with respect to human exposure to radio frequency electromagnetic fields. $300 \mathrm{kHz}$ to $100 \mathrm{GHz}$, ANSI 1992. 
[8] M. Nagai, K. Nakamura, H. Uehara, T. Kanamoto, Y. Takahashi, T. Furukawa, J. Polym. Sci., Part B: Polym. Phys., 37 (1999) 2549-2556.

[9] V.L. Strashilov, J. Appl. Phys., 88 (2000) 3582-3586.

[10] H.S. Nalva, Ferroelectric Polymers, Marcel Dekker Inc, New York, 1955.

[11] P. Sajkiewicz, Eur. Polym. J., 35 (1999) 1581-1590.

[12] K. Nakagawa, Y. Ishida, J. Polym. Sci., Part B: Polym. Phys., 11 (1973) 2153-2171.

[13] D. Tabor, The hardness of Metals, Clarendon Press, Oxford 1951.

[14] F.J.B. Calleja, Adv. Polym. Sci., 66 (1985) 117-148.

[15] A.J. Lovinger, H.D. Keith, Macromolecules, 12 (1979) 919-924.

[16] G.T. Davis, J.E. McKinney, M.G. Broadhurst, S.C. Roth, J. Appl. Phys., 49 (1978) 4998-5002.

[17] A.J. Lovinger, Developments in crystalline polymers-I, Applied Science Publishers, London, 1982.

[18] R. Hasegawa, Y. Takahashi, Y. Chatani, T. H., Polym. J., 3 (1972) 600-610.

[19] M. Kobayashi, K. Tashiro, H. Tadokoro, Macromolecules, 8 (1975) 158-171.

[20] R. Gregorio Jr, R.C. Capitao, J. Mat. Sci., 35 (2000) 299-306.

[21] R. Gregorio Jr, J. Appl. Polym. Sci., 100 (2006) 3272-3279.

[22] R.E. Belke, I. Cabasso, Polymer, 29 (1988) 1831-1842.

[23] T.V. Rao, K.L. Chopra, Thin Solid Films, 60 (1979) 387-393.

[24] W. Wang, S. Zhang, L.O. Srisombat, T.R. Lee, R.C. Advincula, Macromol. Mater. Eng., 296 (2011) 178184.

[25] C. Marega, A. Marigo, Eur. Polym. J., 39 (2003) 1713-1720.

[26] C.G. Vonk, F.J. Baltá-Calleja, X-Ray Scattering of Synthetic Polymers, Elservier Amsterdam, 1989.

[27] A.J. Ryan, J.L. Stanford, W. Bras, T.M.W. Nye, Polymer, 38 (1997) 759-768.

[28] B. Crist, J. Polym. Sci., Part B: Polym. Phys., 39 (2001) 2454-2460.

[29] M.L. Cerrada, R. Benavente, E. Pérez, Macromol. Chem. Phys., 202 (2001) 2686-2695.

[30] J.M. Martínez-Burgos, R. Benavente, E. Pérez, M.L. Cerrada, J. Polym. Sci., Part B: Polym. Phys., 41 (2003) 1244-1255.

[31] V. Lorenzo, J.M. Pereña, J.M.G. Fatou, Angew. Makromol. Chem., 172 (1989) 25-35.

[32] M.L. Cerrada, J.L. De La Fuente, M. Fernández-García, E.L. Madruga, Polymer, 42 (2001) 4647-4655.

[33] J. Arranz-Andres, J.L. Guevara, T. Velilla, R. Quijada, R. Benavente, E. Perez, M.L. Cerrada, Polymer, 46 (2005) 12287-12297. 
[34] M.L. Cerrada, J.L. De la Fuente, E.L. Madruga, M. Fernández-García, Polymer, 43 (2002) 2803-2810.

[35] M.L. Cerrada, M.F. Laguna, R. Benavente, E. Pérez, Polymer, 45 (2004) 171-179.

[36] J.M. Lopez-Majada, H. Palza, J.L. Guevara, R. Quijada, M.C. Martinez, R. Benavente, J.M. Perena, E. Perez, M.L. Cerrada, J. Polym. Sci., Part B: Polym. Phys., 44 (2006) 1253-1267.

[37] J. Joo, A.J. Epstein, Appl. Phys. Lett., 65 (1994) 2278-2280.

[38] N.C. Das, T.K. Chaki, D. Khastgir, A. Chakraborty, J. Appl. Polym. Sci., 80 (2001) 1601-1608.

[39] Y. Yang, M.C. Gupta, K.L. Dudley, R.W. Lawrence, J. Nanosci. Nanotechnol., 7 (2007) 549-554.

[40] B.L. Henke, E.M. Gullikson, J.C. Davis, At. Data Nucl. Data Tables, 54 (1993) 181-342.

[41] J. Arranz-Andrés, E. Pérez, M.L. Cerrada, Eur. Polym. J., 48 (2012) 1160-1168.

[42] P.E. Arranz-Andrés J, Cerrada M L, Europ. Polym. J., 48 (2012) 1160-1168. 


\section{Tables caption}

Table 1. First melting and crystallization temperatures obtained from DSC experiments: $T_{m}$, and $T_{c}$, respectively; as well as normalized enthalpy and crystallinity values: $\Delta H_{m}{ }^{\text {norm }}, f_{c}{ }^{m}, \Delta H_{c}{ }^{\text {norm }}$ and $f_{c}{ }^{c}$, for the first melting and crystallization processes.

Table 2. Mechanical parameters for the different hybrids analyzed at $23^{\circ} \mathrm{C}$ : Young's modulus, E; yield stress, $\sigma_{Y}$; yield strain, $\varepsilon_{Y}$; tensile strength at break, $\sigma_{B}$; deformation at break, $\varepsilon_{B}$ and toughness, $T$.

Table 1.

\begin{tabular}{c|c|c|cccccc}
\hline specimen & $\begin{array}{c}\text { Al } \\
\text { wt.\% }\end{array}$ & $\begin{array}{c}\mathbf{T}_{\mathbf{g}} \\
\left({ }^{\circ} \mathbf{C}\right)\end{array}$ & $\begin{array}{c}\mathbf{T}_{\mathbf{m}} \\
\left({ }^{\circ} \mathbf{C}\right)\end{array}$ & $\begin{array}{c}\Delta \mathbf{H}_{\mathbf{m}}{ }^{\text {norm }} \\
(\mathbf{J} / \mathbf{g})\end{array}$ & $\mathbf{f}_{\mathrm{c}}{ }^{\mathbf{m}}$ & $\begin{array}{c}\mathbf{T}_{\mathrm{c}} \\
\left({ }^{\circ} \mathbf{C}\right)\end{array}$ & $\begin{array}{c}\Delta \mathbf{H}_{\mathrm{c}^{\text {norm }}} \\
(\mathbf{J} / \mathbf{g})\end{array}$ & $\mathbf{f}_{\mathrm{c}}{ }^{\mathbf{c}}$ \\
\hline PVDF & 0.0 & -44.0 & 168.5 & 64.0 & 0.61 & 141.0 & 61.5 & 0.59 \\
Al1 & 1.5 & -44.5 & 168.5 & 64.0 & 0.61 & 141.0 & 66.0 & 0.63 \\
Al2 & 3.1 & -44.5 & 169.5 & 67.5 & 0.64 & 140.5 & 68.0 & 0.65 \\
Al5 & 8.0 & -45.0 & 169.5 & 66.5 & 0.64 & 140.5 & 66.5 & 0.64 \\
Al8 & 13.2 & -45.0 & 169.5 & 65.5 & 0.63 & 141.0 & 62.5 & 0.60 \\
Al10 & 16.9 & -45.0 & 168.0 & 65.5 & 0.63 & 141.0 & 63.5 & 0.61 \\
Al15 & 26.8 & -42.5 & 168.5 & 73.4 & 0.70 & 141.5 & 61.0 & 0.58 \\
Al20 & 37.9 & -45.0 & 168.5 & 77.5 & 0.74 & 141.5 & 59.5 & 0.57 \\
\hline
\end{tabular}


Table 2.

\begin{tabular}{cccccccc}
\hline Specimen & $\begin{array}{c}\mathrm{Al} \\
\% \text { vol. }\end{array}$ & $\begin{array}{c}\mathrm{E} \\
(\mathbf{M P a})\end{array}$ & $\begin{array}{c}\boldsymbol{\sigma}_{\mathrm{Y}} \\
(\mathbf{M P a})\end{array}$ & $\begin{array}{c}\boldsymbol{\varepsilon}_{\mathrm{Y}} \\
(\%)\end{array}$ & $\begin{array}{c}\sigma_{\mathrm{B}} \\
(\mathbf{M P a})\end{array}$ & $\begin{array}{c}\boldsymbol{\varepsilon}_{\mathrm{B}} \\
(\%)\end{array}$ & $\begin{array}{c}\mathbf{T} \\
\left(\mathbf{k J} / \mathbf{m}^{2}\right)\end{array}$ \\
\hline PVDF & 0 & 1250 & 50 & 8 & 35 & 41 & 225 \\
Al1 & 1 & 1280 & 50 & 8 & 34 & 17 & 95 \\
Al2 & 2 & 1350 & 50 & 7 & 36 & 15 & 85 \\
Al5 & 5 & 1540 & 50 & 5 & 31 & 7 & 36 \\
Al8 & 8 & 1630 & 54 & 6 & 42 & 6 & 28 \\
Al10 & 10 & 1650 & 54 & 5 & 36 & 7 & 30 \\
Al15 & 15 & 1990 & 56 & 6 & 54 & 7 & 42 \\
Al20 & 20 & 2210 & 55 & 4 & 50 & 4 & 17 \\
\hline
\end{tabular}




\section{Figure Captions}

Figure 1. Reflection X-ray diffraction patterns, at room temperature, of pristine PVDF and the distinct (nano)composites with different contents of Al nanoparticles.

Figure 2. FTIR spectra, at room temperature, of PVDF and the distinct hybrids with different contents of Al nanoparticles, in two wave number intervals.

Figure 3. SEM pictures of PVDF/AI hybrids with different contents of Al nanoparticles.

Figure 4. a) DSC first melting curves and b) cooling curves for PVDF homopolymer and its Al hybrids. Scanning rate: $20^{\circ} \mathrm{C} / \mathrm{min}$.

Figure 5. Real-time X-ray diffraction profiles for PVDF as function of temperature using synchrotron radiation in a melting experiment at $8{ }^{\circ} \mathrm{C} / \mathrm{min}$ : a) wide-angle and b) small angle patterns, respectively. c) Temperature dependence, on melting, of the SAXS relative invariant and its derivative (upper plot) and on the most probable long spacing (lower plot).

Figure 6. Dependence of microhardness on Al nanoparticles content.

Figure 7. Stress-strain curves for the neat PVDF matrix and the different Al hybrids at room temperature and $10 \mathrm{~mm} \mathrm{~min}^{-1}$.

Figure 8. Variation of the Young's modulus with the Al nanoparticles content. In the insert: Relationship between microhardness, $\mathrm{MH}$, and elastic modulus in the pristine PVDF polymer and the nanocomposites. Figure 9. Transmission 2D X-ray photograph for PVDF (left) and Al15 hybrid (right).

Figure 10. Variation with the Al nanoparticles content of X-ray intensity reaching CCD, normalized to a thickness of $1 \mathrm{~mm}$ in the different hybrids.

Figure 11. Dependence of EMI shielding upon Al composition for the different hybrid materials analyzed. 


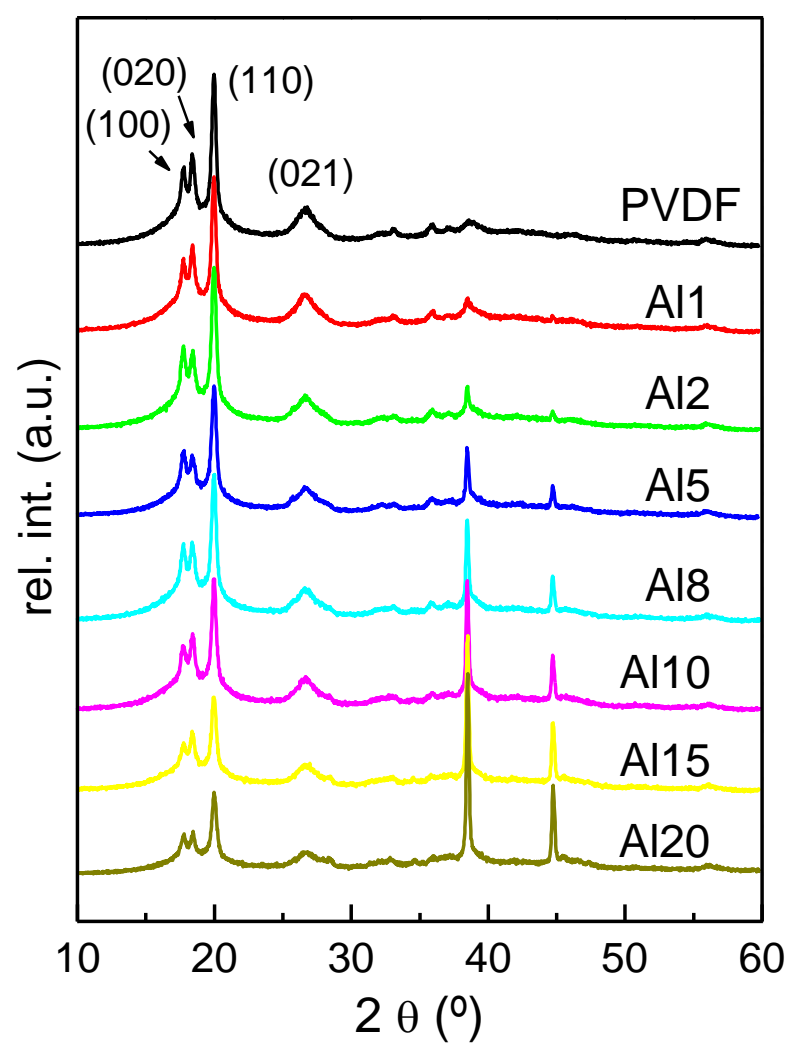

Figure 1. 

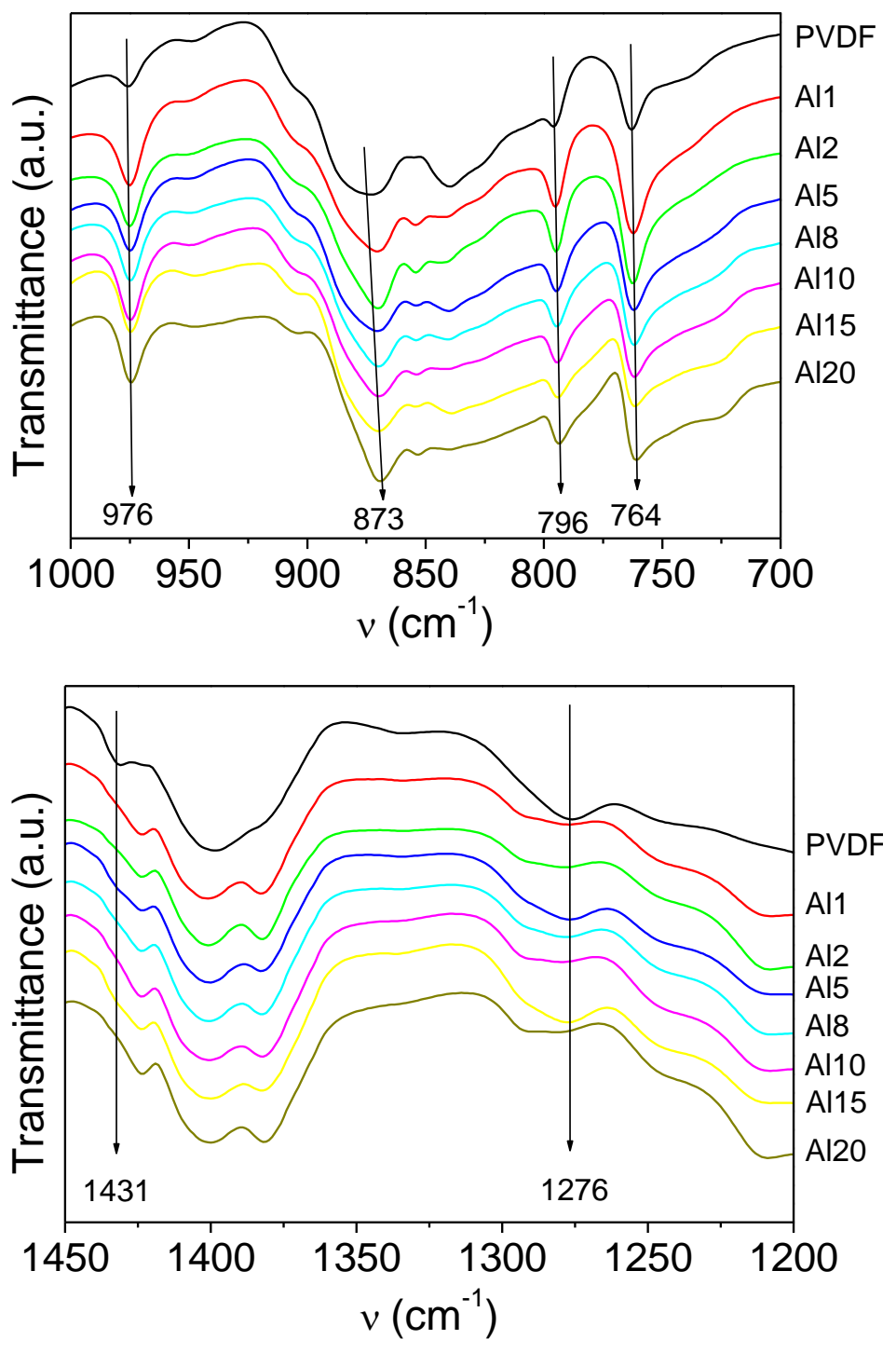

Figure 2. 


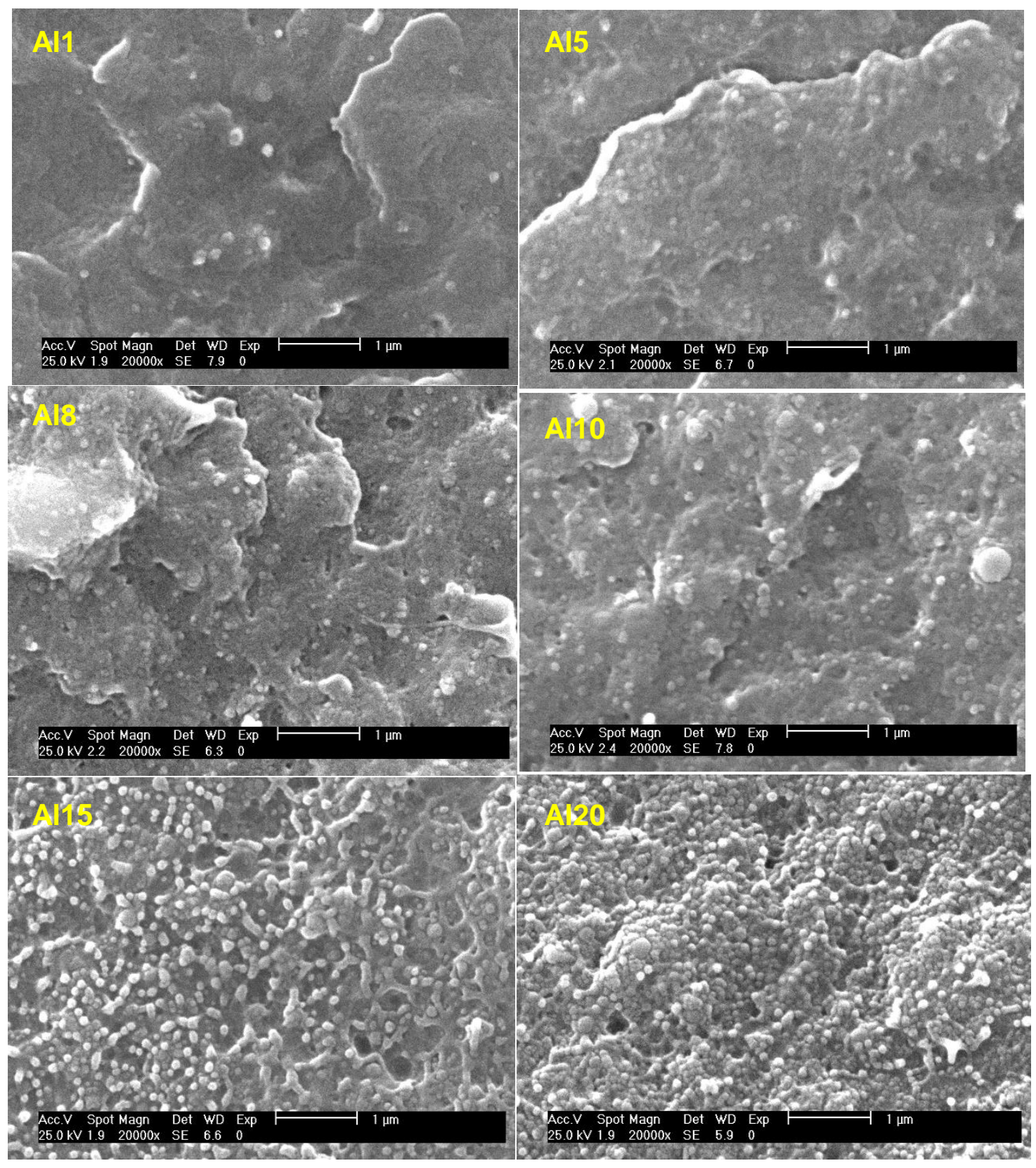

Figure 3. 

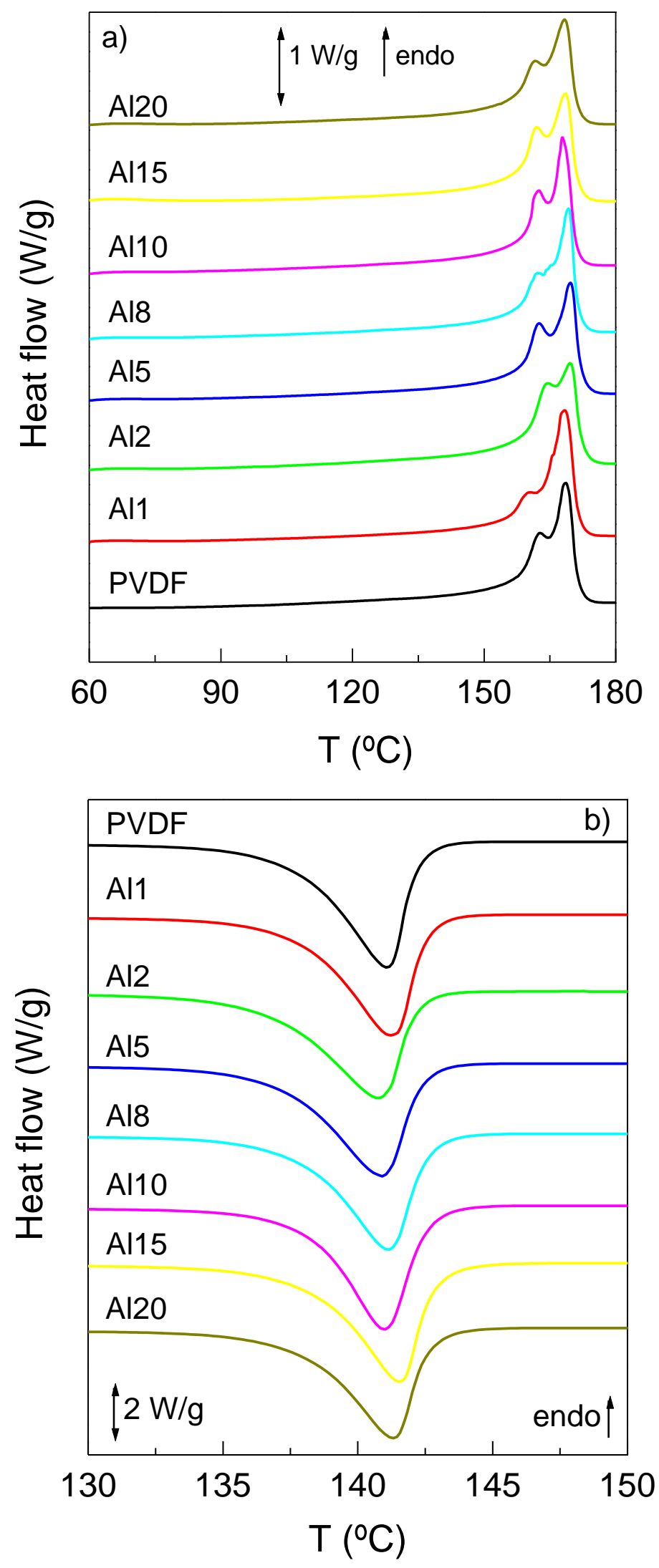

Figure 4. 


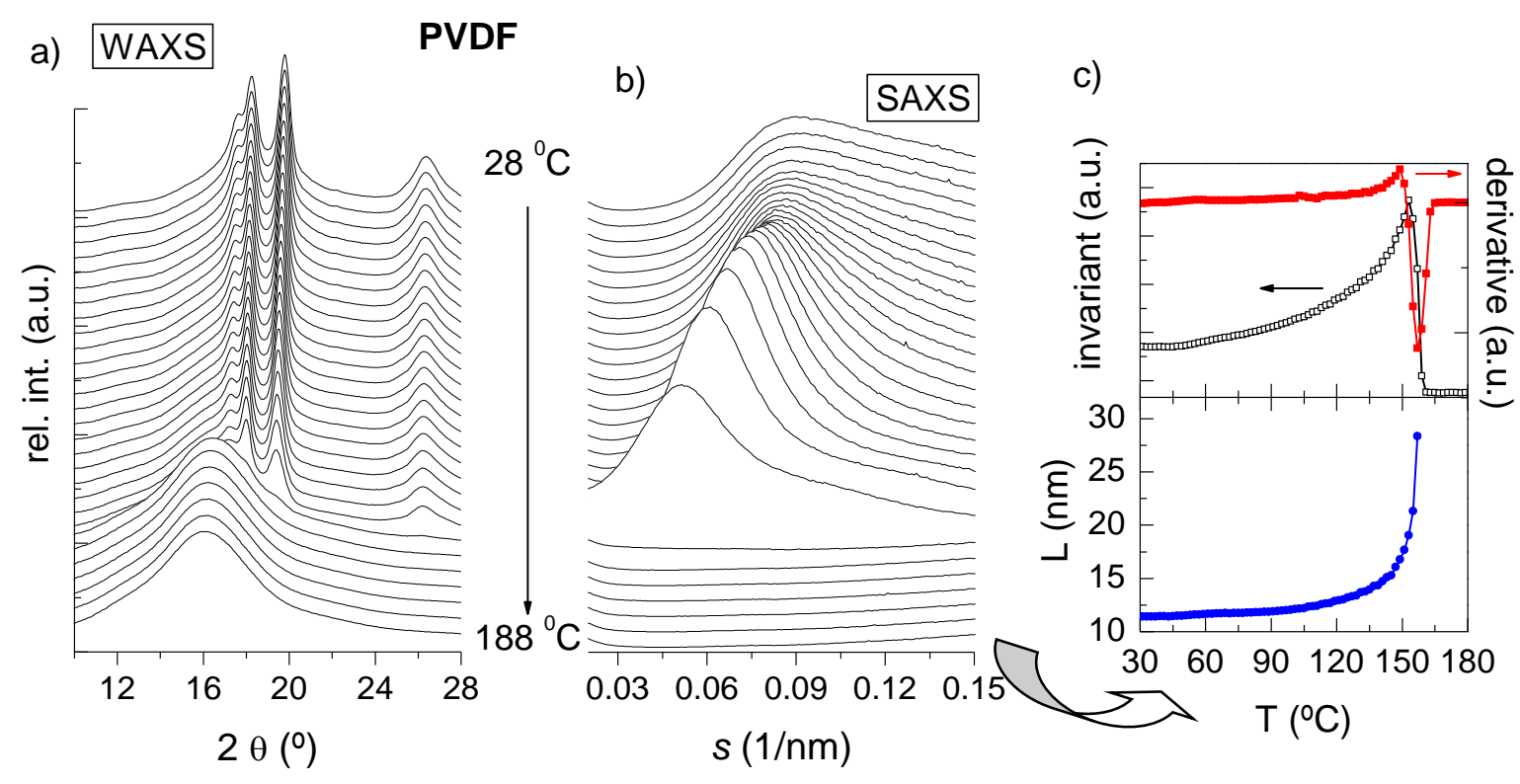

Figure 5. 


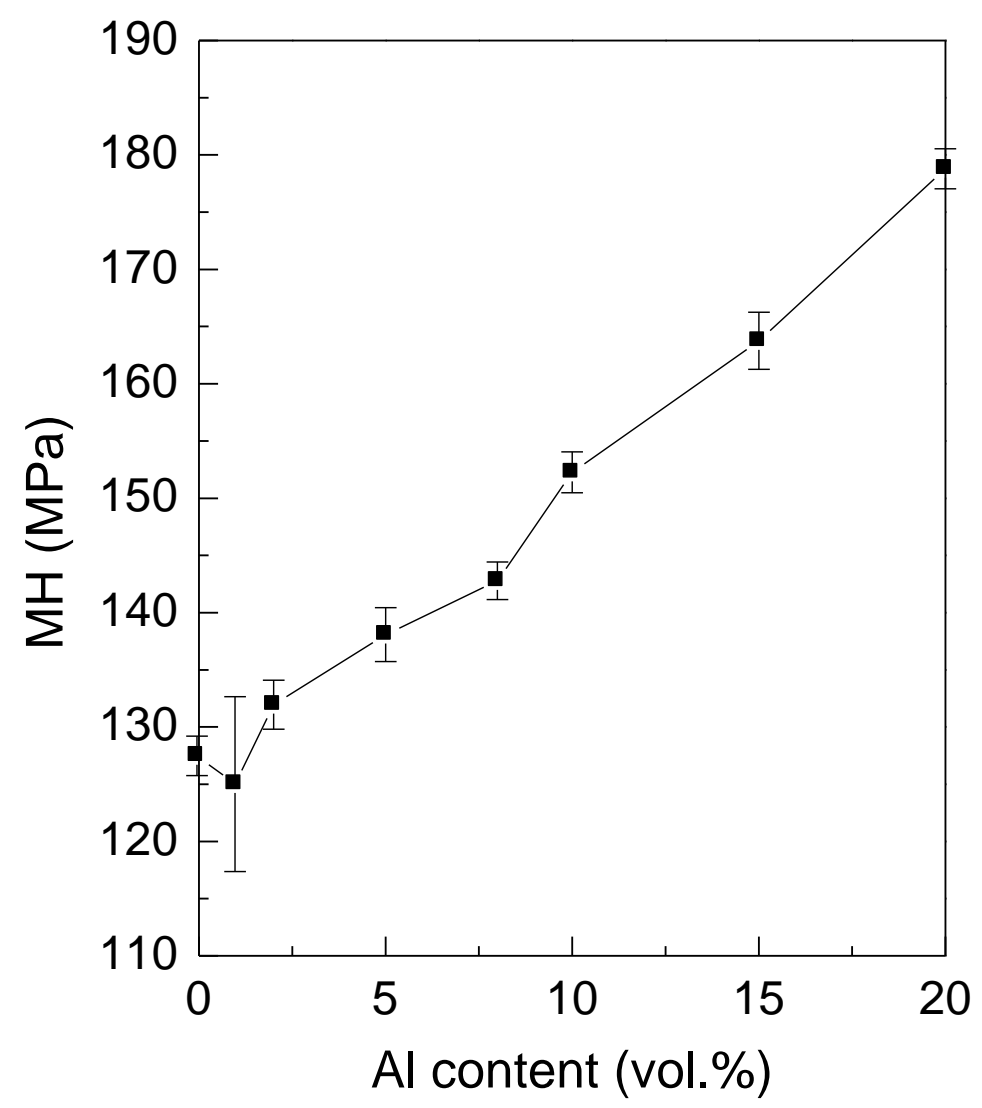

Figure 6. 


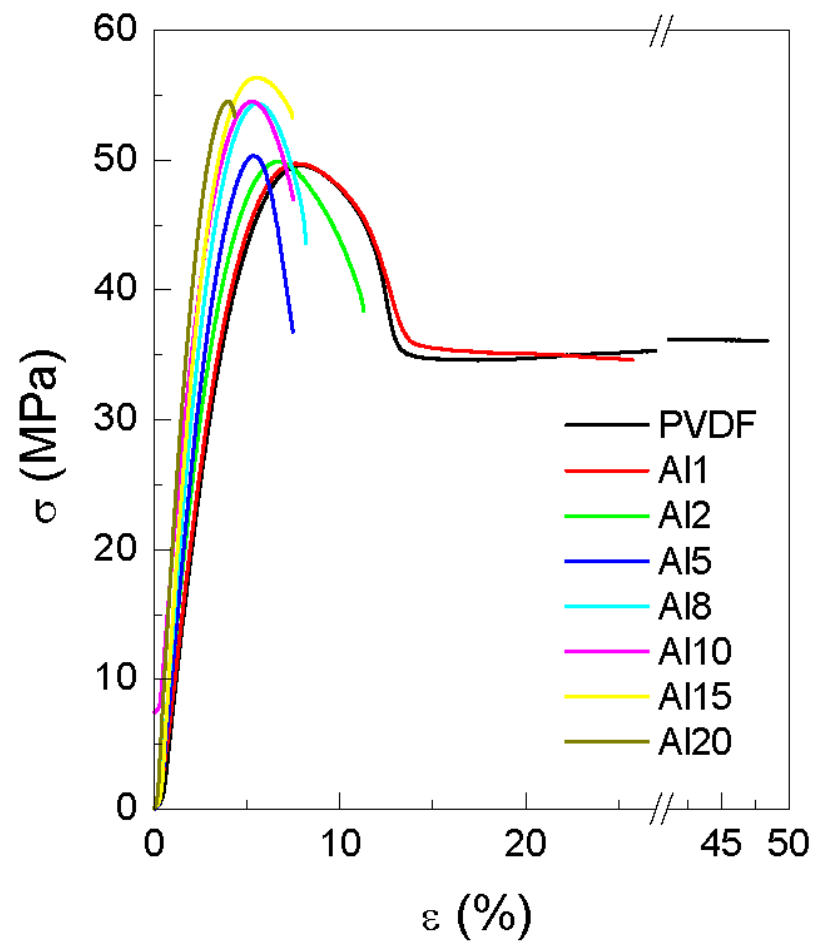

Figure 7. 


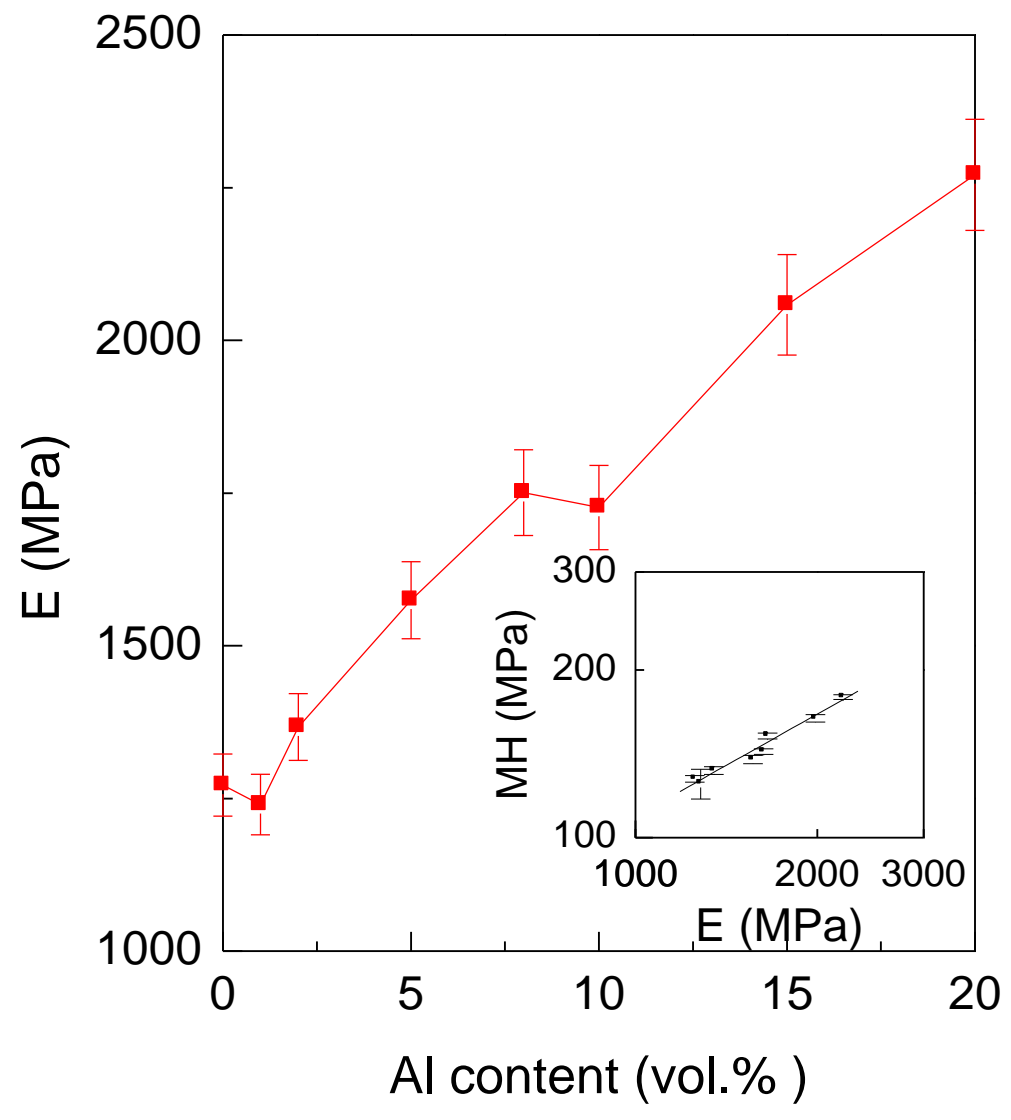

Figure 8. 


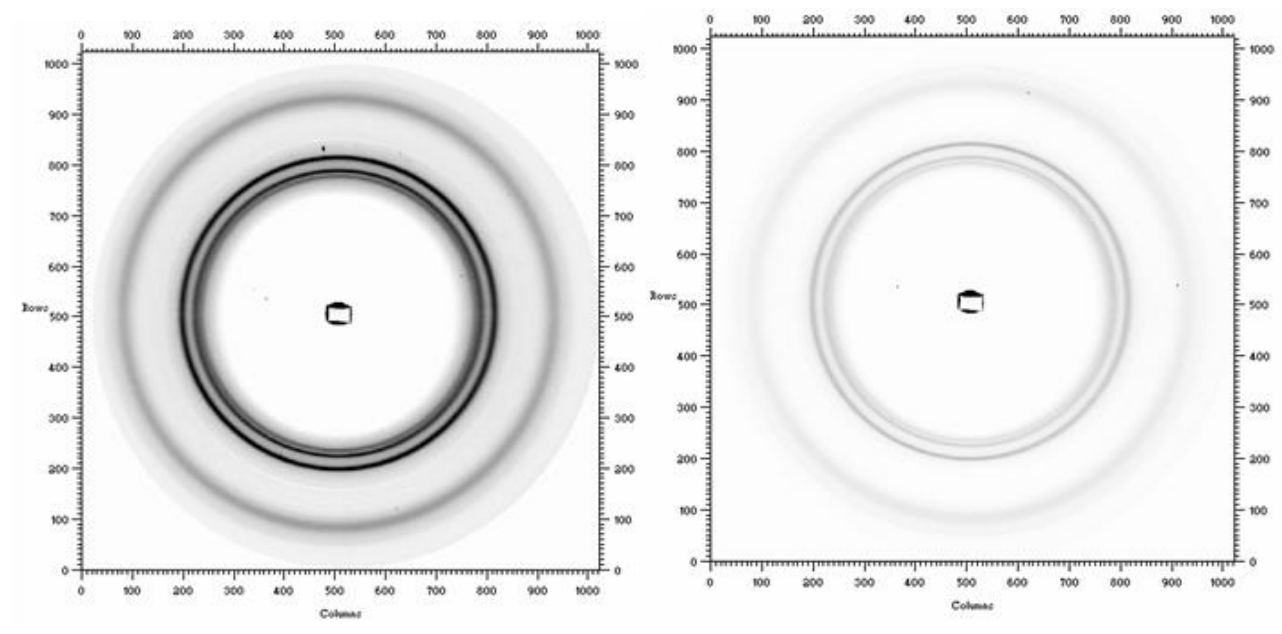

Figure 9. 


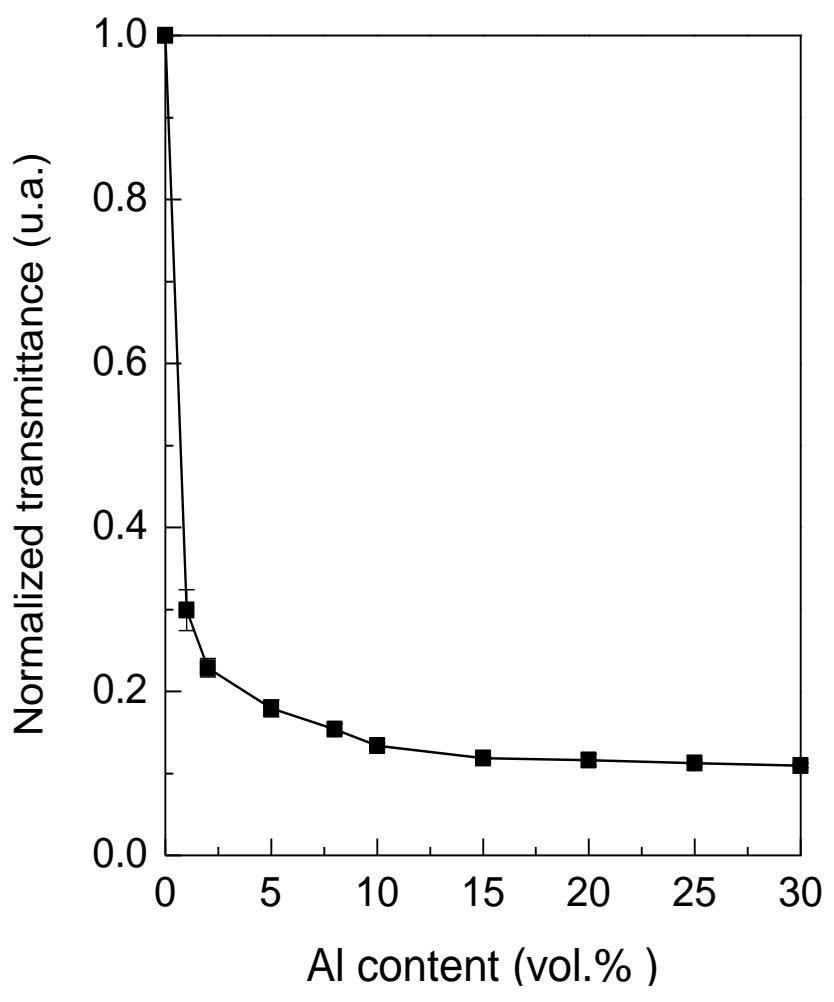

Figure 10. 


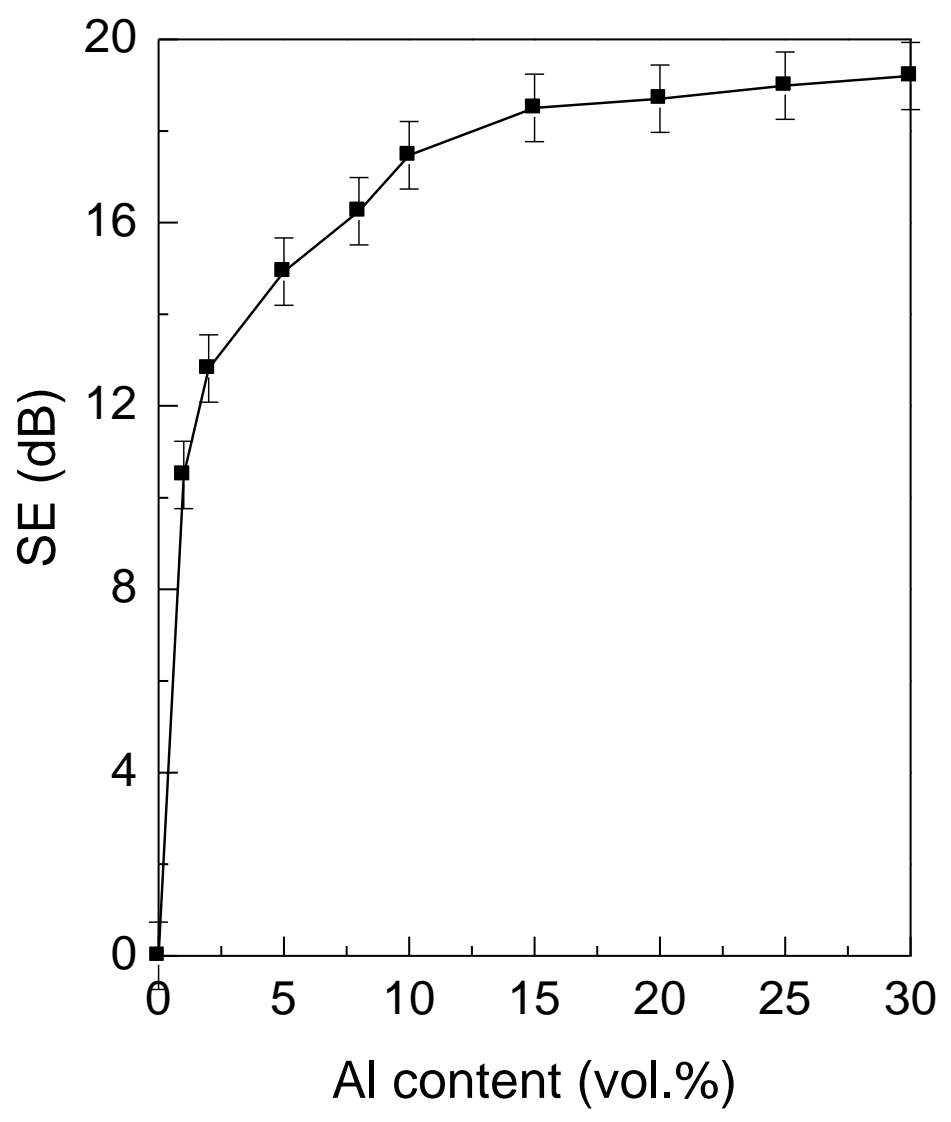

Figure 11. 